\title{
Autocorrelation Based White Space Detection in Energy Harvesting Cognitive Radio Network
}

\author{
Samson I. Ojo, Zachaues K. Adeyemo, Rebecca O. Omowaiye and Oluwatobi O. Oyedokun \\ Electronic and Electrical Engineering Department, Ladoke Akintola University of Technology, Ogbomoso, Oyo \\ State, Nigeria
}

\begin{tabular}{l}
\hline \hline Article Info \\
\hline Article history: \\
Received Jun 16, 2021 \\
Revised Oct 21, 2021 \\
Accepted Nov 9, 2021 \\
\hline
\end{tabular}

\section{Keywords:}

White Space

Antenna Switching

Secondary User

Primary User

RF energy harvesting

\begin{abstract}
Accurate detection of White Space (WS) is of paramount importance in a Cognitive Radio Network (CRN) to prevent authorized users from harmful interference. However, channel impairment such as multipath fading and shadowing affects accurate detection of WS resulting in interference. The Existing Feature Detection (EFD) technique used to address the problem is faced with computational complexity and synchronization resulting in long sensing time, bandwidth inefficiency, energy constrain and poor detection rate. Hence, this paper proposes autocorrelation based multiple antenna with energy harvesting for WS detection in a CRN using Radio Frequency (RF) energy harvesting and autocorrelation of the received signal with a modified Equal Gain Combiner (mEGC). Antenna Switching (AS) RF energy harvesting with mEGC are used to harvest energy and information from the received PU signal in a multiple antenna configuration. Autocorrelation is then obtained and compared with the set threshold of zero to determine the presence or absence of WS. The proposed technique is evaluated using Spectral Efficiency (SE), Probability of Detection (PD) and Sensing Time (ST) by comparing with EFD technique. The results obtained revealed that the proposed technique shows better performance than EFD.
\end{abstract}

Copyright $(52021$ Institute of Advanced Engineering and Science. All rights reserved.

\section{Corresponding Author:}

Adeyemo, Zachaeus K.

Department of Electronic and Electrical Engineering,

Ladoke Akintola University of Technology, Ogbomoso, Oyo State, Nigeria

Email: zkadeyemo@lautech.edu.ng

\section{INTRODUCTION}

The extensive increase in the demand of wireless communication services due to their functions in many facets of human endeavor has led to exponential increase in the demand of radio spectrum. The rapid increase in the requirement of radio spectrum to meet up with the extensive growth in wireless communication result in shortage of spectrum. The available radio spectrum is limited and getting crowded day by day as the number of users accessing wireless communication service increases [1-3]. The research conducted on availability of radio spectrum revealed that, the shortage of spectrum is not only due to insufficient spectrum but also to the traditionally fixed spectrum access preventing other parties from using the allocated spectrum rather than authorized user. However, many portions of the assigned spectrum are not used during significant period of time and average usage of many allocated spectrum is below $15 \%[4,5]$. Therefore, unavailability of radio spectrum which is one of the major problems of wireless communication is due to underutilization of the assigned spectrum. Since the regulatory process of the new radio spectrum is time consuming and cost ineffective, the efficient usage of allocated spectrum is of paramount importance in solving the problem of spectrum scarcity in wireless communication [6-8]. Cognitive Radio Network (CRN) is proposed to effectively use the available spectrum by allowing unauthorized user to exploit the allocated spectrum when it becomes idle. CRN is a communication technique in which an unauthorized user known as Secondary User (SU) detects the assigned spectrum that is busy or idle. This, then, instantly allows transmitting terminals to 
opportunistically make use of idle spectrum while avoiding the occupied ones. It is a technology that advocates the concept of dynamic spectrum access, contrary to the existing fixed spectrum access [9-11]. CRN improves spectrum utilization by allowing unauthorized users to access the allocated spectrum without interfering with the authorized user, that is, Primary User (PU). The performance of a CRN depends on accurate detection of White Space (WS) otherwise known as spectrum hole, before allowing transmitting terminals to opportunistically make use of idle spectrum. This is achieved through Spectrum Sensing (SS) which is the process by which unauthorized user senses the assigned spectrum over a certain frequency band to detect unused spectrum known as WS [12-14].

Therefore, SS is one of the major operations in CRN and can be achieved by using either single antenna or multiple antenna configuration. In multiple antenna configuration, single antenna is used by the licensed user to transmit signal while, multiple antenna is used at the SU. On the other hand, single antenna SS involves the use of only one antenna at the both users. Multiple antenna system performs relatively better than single antenna system due to increase in an authorized signal strength but requires a diversity combiner to combine the multiple copies of PU signal. [15-17]. Cyclostationary Detector (CD) is one of detectors that commonly used to detect the presence or absence of PU signal due to its high detection rate even at a very low PU signal strength. CD detects signal by capturing the periodicity of statistical parameters using different features extraction such as windowing and theory of Hilbert transform [18]. However, CD requires synchronization between PU and SU due to prior knowledge about PU signals, which is very difficult to achieve in practice. Also, the detector suffers from high computational complexity due to windowing and Hilbert transform used to extract the cyclostationary features of PU signal resulting in long sensing time as well as high power consumption. Wireless communication system requires energy efficiency to increase the revenue of service provider by reducing operational expenditure through saving on the electricity bills thereby reducing the joule per bit cost that keeps mobile services affordable for the users. Multiple antenna CRN suffers from energy constrain due to diversity combiner involved before carrying out SS. Radio Frequency Energy Harvesting (RFEH) which is the process of converting the energy presence in the RF signal into electrical energy for in powering a node is incorporated into multiple antenna CRN to solve the problem of energy constrain. Antenna Switching (AS) RFEH is adopted in this paper due to its ability to harvest energy and information simultaneously. Equal Gain Combiner (EGC) with single RF chain and Match Filter (MF) is used to combine the multiple copies of the received licensed user signal before carrying out energy and information harvesting simultaneously [19-20]. The sensing accuracy, which is the ability of SU to sense PU without interference, depends on fading channel of PU and SU. Many authors such as [1-2], [21] have worked on SS in CRN using Rayleigh, Nakagami and Log-Normal fading distributions which are good for terrestrial modelling. However, Rayleigh and Nakagami can only be used in modelling multipath fading while Lognormal can only be used in modelling shadowing. Moreso, in multiple antenna SS, the combined effects of multipath fading and shadowing are present. Therefore, in this paper, combined Rayleigh and Log-Normal fading distributions are considered due to its ability to model multipath fading and shadowing simultaneously.

There have been various existing works on white space detection using cyclostationary features detector for detection of WS in a multiple antenna CR. Authors in [18] proposed a spectrum sensing technique in CR based on features detection using windowing approach to address problem of noise uncertainty in Energy Detector (ED) leading to poor detection rate. Cyclostationary features of the received signal were extracted using hamming windowing. The extracted features were then compared with the known features of PU signal and if the features are the same, the decision is that, PU is present, otherwise PU is absent. The results obtained revealed that, the proposed technique overcomes the problem of noise uncertainty in ED. However, the technique suffers from computational complexity and high bandwidth consumption due to windowing and synchronization used. Cyclostationary feature detection-based spectrum sensing algorithm under complicated electromagnetic environment in CR networks was carried out in [6] to solve the problem of noise uncertainty during SS in a CR networks. In this paper, feature extraction was carried out on the received signal using Hilbert transform theory. The features such as modulation scheme and coding extracted were compared with the known feature of PU signal. Authors stated that, if the feature extracted is the same with known feature of PU signal, the decision is that PU is present, otherwise PU is absent. The results obtained confirmed better performance of the technique when compared with ED but suffers from long sensing time and high bandwidth consumption due to Hilbert transform theory and synchronization required, respectively. Also, feature detection based blind approach for SS and classification technique was proposed in [22] to solve the problem of harmful interference in a CR network. In this technique, unauthorized multiple antenna received the multiple copies of authorized user's signal over Rayleigh fading channel. The cyclostationary features of the received signals were then obtained using neural networks and compared with a known PU signal features to make a decision on the idleness of the spectrum. The results obtained revealed that, the technique has a better performance with high detection rate when compared with ED. However, the technique has computational 
complexity and high bandwidth consumption due to synchronization between PU signal and SU, and this makes the technique to be difficult to implement in practice.

Furthermore, Maritime CR spectrum sensing based on multiple antenna feature detection was proposed in [23] for accurate detection of white space in CRN. Problem of noise uncertainty during SS that resulting in harmful interference in CRN was addressed using periodicity of PU signal. The periodicity of the received PU signa was obtained using black man windowing and compared with known periodicity of transmitted PU signal. Authors stated that, if the obtained periodicity is the same as the PU periodicity, then there is absence of white space, otherwise white space is present. The accuracy of detection rate of this technique is far better than that of ED but the technique suffered from computational complexity due to black man windowing used resulting in long sensing time. Also, the technique requires synchronization between the PU signal and SU for periodicity comparison leading to high bandwidth consumption and the technique is difficult to implement in practice due to synchronization required. The contributions of this paper are as follows:

1) proposes an energy efficient multiple antenna white space detection that reduces operational expenditure thereby making the services affordable to every user. This is achieved by using RF energy harvesting to power the licensed user node during spectrum sensing, while reserving its own energy for signal transmission. Also, the existing works on CD suffer from computational complexity due to windowing and Hilbert transform used to extract the cyclostationary feature of PU signal. The propose work makes use of autocorrelation function of the received PU signal, thereby reducing the computational complexity that results in high power consumption.

2) derives a closed form expression for Probability Density Function (PDF) of combined Rayleigh and Lognormal fading channel distribution.

3 ) the existing works on white space detection were based on single fading distribution which can only model either multipath fading or shadowing effect. However, in multiple antenna spectrum sensing, the two impairments occurred simultaneously. In this paper, the detection of white space is based on the combined Rayleigh and Log-normal fading distribution that can model the two major impairments, that is, multipath fading and shadowing effects.

4) the existing CD suffered from high bandwidth consumption due to synchronization between licensed and unlicensed user signals. This synchronization is also difficult to achieve in practice. The proposed technique makes use of autocorrelation of the received signal and a set threshold of zero. Therefore, synchronization that consumes bandwidth is not efficient to implement in practice.

The remaining paper is organized as follows; the proposed white space detection technique is presented in section 2. In this section, the PDF of the combined fading channel and autocorrelation of the received signal are derived. Also, the RF energy harvesting is carried out in this section. Section 3 presents the simulation results as well as performance comparison, while section 4 concludes the paper.

\section{PROPOSED WHITE SPACE DETECTION TECHNIQUE}

The Probability Density Function (PDF) of the combined Rayleigh and Log-Normal fading channel is obtained by multiplying the PDF of distributions. Figure 1 shows the effects of multipath fading and shadowing that occur simultaneously during spectrum sensing. Thus, indicates the suitability of the combined Rayleigh and Log-Normal fading channel to model white space detection technique in a multiple antenna CR. The detection of white space over combined fading channels is carried out using multiple antenna at the SU node, modified Equal Gain Combiner (mEGC) and autocorrelation of the combined signal. The mEGC makes use of single RF chain and matched filter, unlike the conventional EGC with many multiple RF chains and matched filters. The purpose of using mEGC in this proposed technique is to reduce hardware complexity, thereby reducing the sensing time as well as power consumption of the proposed technique. Autocorrelation of the combined signal is obtained and used as a test statistic to determine the presence or absence of PU signal. The proposed technique is simulated using MATLAB R2018a to investigate the performance of the proposed technique. The simulation is carried out over different paths ' $L$ ' $(2,3,4)$ to investigate the effect of number of paths on the proposed technique due to the nature of urban environment. 


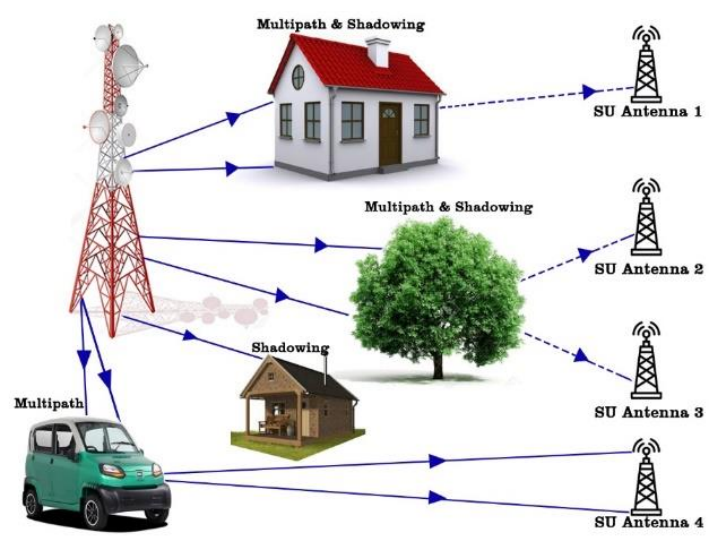

Figure 1. Effect of Multipath Fading and Shadowing in a Multiple Antenna SS

\subsection{Combined Fading Channel}

The combined Rayleigh and Log-Normal fading distributions is considered for the proposed technique as shown in Figure 2, $h_{1}$ and $h_{2}$ represent Rayleigh and Log-Normal fading distributions, respectively. The combined fading distribution is used in the modelling and evaluating of the performance of the proposed technique. The Probability Density Function (PDF) of combined fading distribution ' $P_{R L}(r)$ ' is given as

$$
P_{R L}(r)=\int_{0}^{\infty} P_{R}(r) \cdot P_{L}(r) d r
$$

where: $P_{R}(r)$ is the PDF of Rayleigh fading distribution

$$
P_{L}(r) \text { is the PDF of Log-Normal fading distribution }
$$

Using the PDF of Rayleigh and Log-Normal distributions, the expression for the combined fading distribution for the proposed technique is given as

$$
P_{R L}(r)=\int_{0}^{\infty} \frac{r}{\sigma^{2}} \exp -\left(\frac{r^{2}}{2 \sigma^{2}}\right) \frac{10 / \ln 10}{r \sigma(2 \pi)^{\frac{1}{2}}} \exp \left(-\frac{(\ln r-\mu)^{2}}{2 \sigma^{2}}\right) d r
$$

where: $r$ is the received signal envelope,

$\sigma$ is the root mean square value of the received signal,

$\mu$ is the mean of $\ln r$.

By solving Equation (2), the closed form expression of the $P_{R L}(r)$ gives

$$
\begin{aligned}
& P_{R L}(r)=\frac{1.733}{\sigma^{3}}\left(\exp \left(-\frac{\mu}{2 \sigma^{2}}\right) \sigma^{3}\left(\frac{\pi}{2}\right)^{1 / 2}\right) \\
& P_{R L}(r)=\frac{1.733}{\sigma^{3}}\left(\exp \left(-\frac{\mu}{2 \sigma^{2}}\right) \sigma^{3}\left(\frac{(2 \pi)^{1 / 2}}{2}\right)\right) \\
& P_{R L}(r)=\frac{1.733}{\sigma^{3}}\left(\exp \left(-\frac{\mu}{2 \sigma^{2}}\right) \sigma^{3}\left(\frac{(2 \pi)^{1 / 2}}{2}\right)\right) \\
& P_{R L}(r)=\frac{1.733}{2}\left(\exp \left(-\frac{\mu^{2}}{2 \sigma^{2}}\right)(2 \pi)^{1 / 2}\right) \\
& P_{R L}(r)=0.8665\left((2 \pi)^{1 / 2} \exp \left(-\frac{\mu^{2}}{2 \sigma^{2}}\right)\right)
\end{aligned}
$$




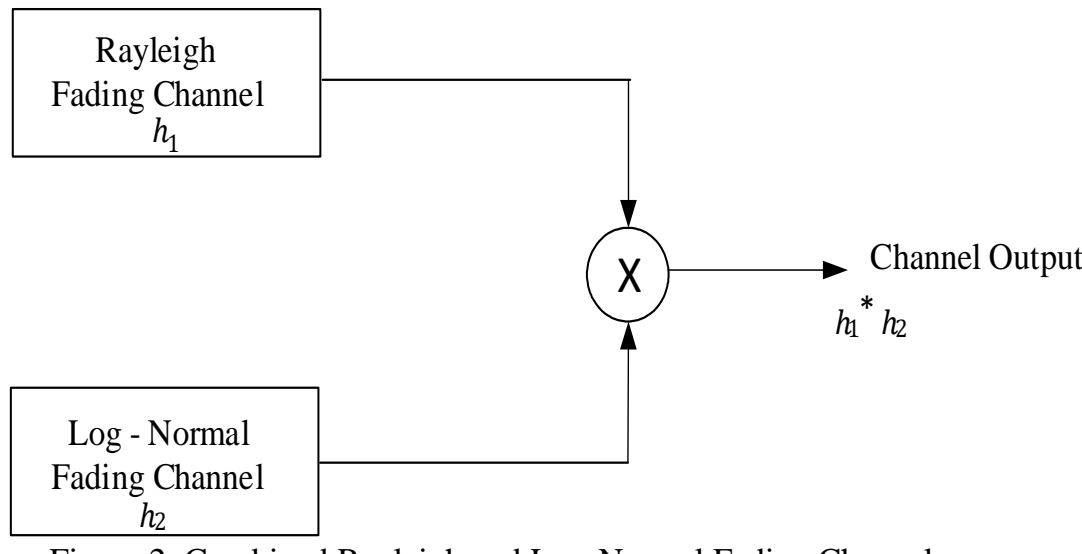

Figure 2. Combined Rayleigh and Log-Normal Fading Channel

\subsection{White Space Detection using Autocorrelation}

Conventional Features Detector (CFD) suffers from computational complexity due to windowing and Hilbert transform technique used in extracting the cyclostationay feature of PU signal. The resulting effect of this computational complexity is long sensing time and high-power consumption. Therefore, in this paper, the cyclostationary feature of PU signal is extracted using the similarities between the signal and its shifted version known as autocorrelation. [6] established the fact that, all the modulated signals are periodic, therefore, PU signal is always a periodic signal while noise is aperiodic signal. Based on this fact, the autocorrelation of the received signal is determined and compared with the set threshold of zero. The multiple copies of the received authorized user's signal over combined Rayleigh and Log-Normal fading channel are received by unauthorized user's multiple antennas and combined at RF stage using Equal Gain Combiner with single RF chain and matched filter (mEGC). Signal output of mEGC is shifted by time $\tau$ and similarities between the signal and its shifted version is determined using autocorrelation function as shown in Figure 3. The output of autocorrelation is then compared with the set threshold of zero to decide whether PU signal exists or not. If the output of autocorrelation is greater than the set threshold, the decision indicates the presence of PU signal due to its ongoing transmission, otherwise PU signal is idle. The autocorrelation function " $R(\tau)$ " of output signal for mEGC is given as

$$
R(\tau)=\int_{-V}^{V} g(t) \times g(t-\tau) d v
$$

where: $\mathrm{v}$ is the period of oscillation $g(t)$ is the received signal which is the output of mEGC $g(t-\tau)$ is the shifted version of the received signal

However, the output signal of mEGC is given as

$$
g(t)_{m E G C}=\frac{1}{w L}\left(\sum_{i=1}^{L} r(i)\right)^{2}
$$

where: $r(i)$ is the signal power on each branch

$\mathrm{L}$ is the number of branches $\mathrm{w}$ is the noise present on each branch

Therefore, substituting Equation (8) into (7) gives

$$
R(\tau)=\frac{1}{(w L)^{2}} \int_{-V}^{V}\left(\sum_{t=1}^{L} g(t)\right)^{2} \times\left(\sum_{t=1}^{L} g(t-\tau)\right)^{2} d v
$$

The detection of idle spectrum known as white space is then depends on Equation (9). If the autocorrelation function of the received signal obtained at any instant is not equal to zero, the decision is WS is not present due to ongoing transmission of PU, otherwise WS is present, that is, the spectrum is idle. This decision is based on the fact that, the autocorrelation of aperiodic signal is always zero, while that of periodic signal is non-zero. Probability of Detection (PD), Spectral Efficiency (SE) and Sensing Time (ST) are the performance metrics used to evaluate the performance of the proposed technique.

\section{a. Detection Rate for the Proposed Technique}

Detection rate which is Probability of Detection (PD) describes the chances of making the right decision on the presence of WS and the higher the value of PD the better the technique. Therefore, to determine the PD, 
the autocorrelation of the received signal is obtained using equation (9) and compared with the set threshold of zero. If the autocorrelation obtained is not equal to zero, then, WS is not present, otherwise the spectrum is idle. Therefore, in this paper, the PD for the proposed technique ' $P D_{A u t}$ ' is given as

$$
P D_{\text {Aut }}=\operatorname{Pr}(R(\tau) \neq 0)
$$

\section{b. Spectral Efficiency (SE) of the Proposed Technique}

The Spectral Efficiency 'SE' which describes the bandwidth efficiency of a technique is given by [24] as

$$
S E=\frac{C}{B}
$$

where: $\mathrm{c}$ is the channel throughput

$\mathrm{B}$ is the bandwidth

But the channel throughput ' $C$ ' is given by [25] as

$$
C=B \log _{2}(1+S N R)
$$

In this paper, the SNR of the received signal is the output of mEGC given in Equation (8)

Therefore, substituting equation (8) into (12) yields

$$
C=B \log _{2}\left(1+\frac{1}{w L}\left(\sum_{i=1}^{L} r(i)\right)^{2}\right)
$$

Using equations (11) and (13), the spectral efficiency for the proposed white space detection technique is obtained as

$$
\begin{aligned}
& S E=\log _{2}\left(1+\frac{1}{w L}\left(\sum_{i=1}^{L} r(i)\right)^{2}\right) \\
& S E=\log _{2}(1)+\log _{2}\left(\frac{1}{w L}\left(\sum_{i=1}^{L} r(i)\right)^{2}\right) \\
& S E=\log _{2}\left(\frac{1}{w L}\left(\sum_{i=1}^{L} r(i)\right)^{2}\right)
\end{aligned}
$$

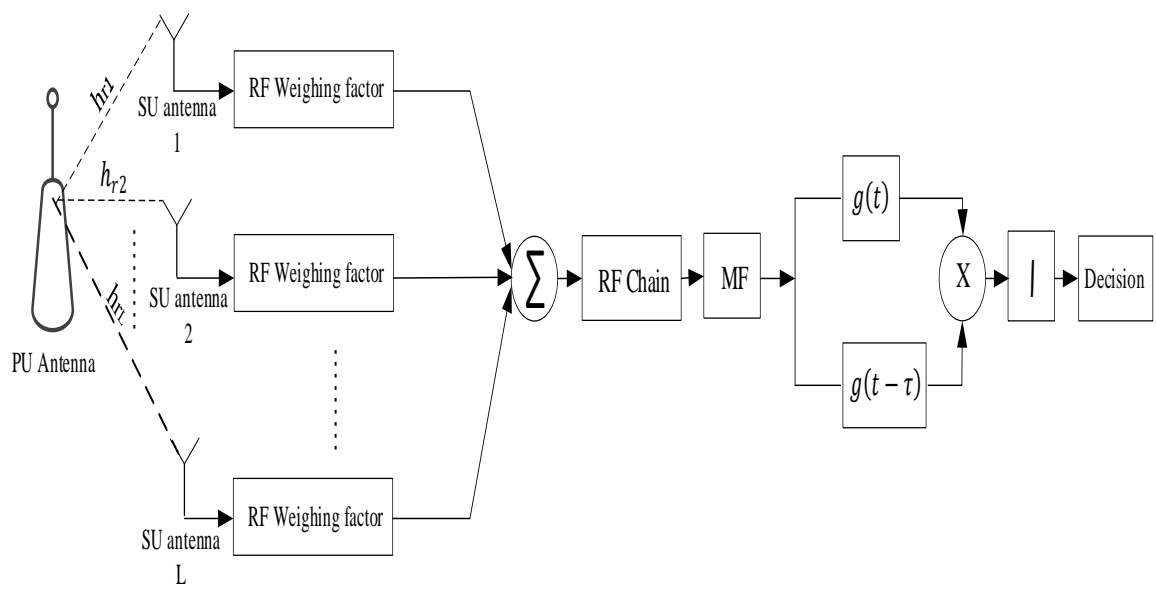

Figure 3. Block diagram of autocorrelation-based spectrum sensing technique

\subsection{Radio Frequency (RF) Energy Harvesting for the proposed technique}

Antenna Switching (AS) RF energy harvesting is used in this paper due to its ability to obtain energy and information from the licensed user signal simultaneously. The multiple copies of the authorized user's signals are received by unauthorized user's antennas and combined using mEGC. The output of the combiner being Alternating Current (AC) voltage is converted to Direct Current (DC) voltage using full wave rectification. The DC voltage is used to charge battery and used as power supply for unlicensed user during spectrum sensing. The purpose of RF energy harvesting is to reserve the unauthorized user energy for transmission while making use of authorized user RF energy during sensing. The randomly generated multiple copies of PU signal through combined Rayleigh and Log-Normal fading channel are received by ' $M$ ' SU 
antennas. The SU antennas are divided into two fractions and the first fraction is used to harvest information for white space detection, while other fraction is used for energy harvesting. The authorized user's signal received by the first set of SU antenna is combined using mEGC and output of the combiner is used for spectrum sensing as shown in Figure 4. The licensed user's signal received by other fractions of SU antenna is also combined using mEGC and the output of combiner is used as input to Impedance Matching (IM) to minimize signal reflection. Output of IM is used as input to voltage multiplier to increase the voltage of the received signal and rectified using a bridge rectifier. The Direct Current (DC) output of the rectifier is filtered using capacitor and regulate using a voltage regulator as shown in Figure 4. DC output of the regulator can then be used to recharge the battery to be used by SU during spectrum sensing period.

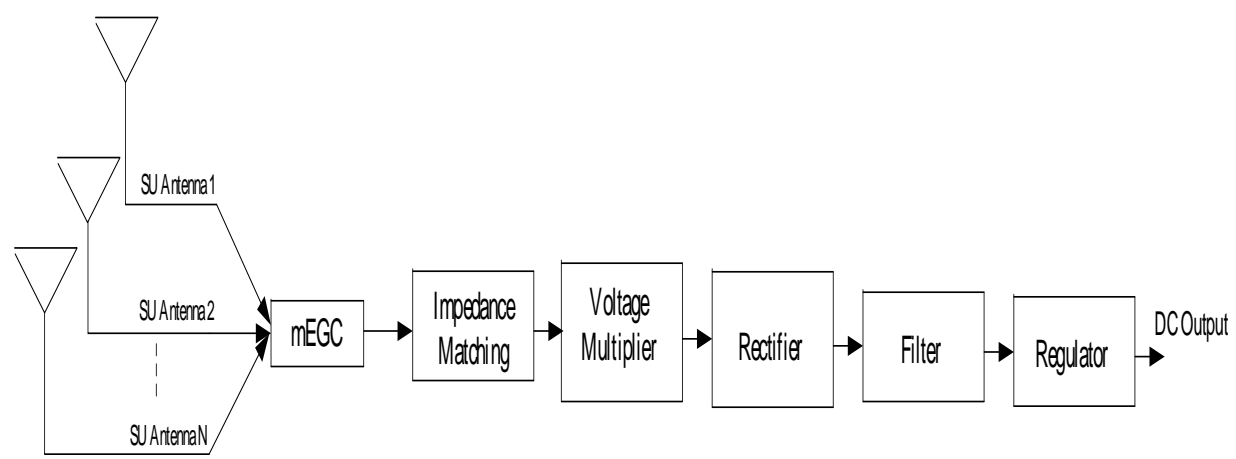

Figure 4. Block Diagram of Energy Harvesting for the proposed technique

\section{SIMULATION RESULTS}

$\mathrm{PD}, \mathrm{SE}$ and ST are the metrics used to evaluate the performance of the proposed technique over combined Rayleigh and log-normal fading channel. The PD, SE and ST values at different SNR with different number of propagation paths were obtained and compared with [23]. Figures 5 to 7 show PD versus SNRs for Proposed Features Detection (PFD) and Existing Features Detection (EFD) over the combined Rayleigh and Log-Normal fading channel at different number of paths. Figure 5 presents PD versus SNRs for the proposed and existing techniques at $\mathrm{L}$ of 2 . At SNR of $4 \mathrm{~dB}$, PD values of 0.4878 and 0.4335 are obtained for PFD and EFD, respectively, while at SNR of $10 \mathrm{~dB}$, PD values of 0.5479 and 0.4872 are obtained for PFD and EFD, respectively. The two techniques show an increase in detection rate at SNR of $16 \mathrm{~dB}$, with PD values of 0.6095 and 0.5591 for PFD and EFD, respectively. The results obtained reveal that the proposed technique has higher detection rate than the existing technique at all the SNR considered due to the combining nature of the mEGC used for the proposed technique that reduces variability in PU signal strength. Figure 6 depicts PD versus SNR at $\mathrm{L}$ of 3 for PFD and EFD. At SNR $10 \mathrm{~dB}$, PD values of 0.5825 and 0.5215 are obtained for PFD and EFD, respectively, while 0.7320 and 0.6715 are the corresponding PD values obtained at SNR of $16 \mathrm{~dB}$. The PD values obtained versus SNR at L of 4 for PFD and EFD are presented in Figure 7. At SNR of $10 \mathrm{~dB}, \mathrm{PD}$ values of 0.7979 and 0.7089 are obtained for PFD and EFD, respectively, while, the corresponding PD values of 0.8878 and 0.8149 are obtained for PFD and EFD, respectively at SNR of $16 \mathrm{~dB}$. Figures 5 to 7 reveal that the proposed technique shows better performance with highest detection rate when compared with EFD due to combining nature of the PU signals in the proposed technique before obtaining autocorrelation. The results obtained also reveal that for all the two techniques, PD increases as number of paths increases and this is due to high significant of autocorrelation as signal strength increases.

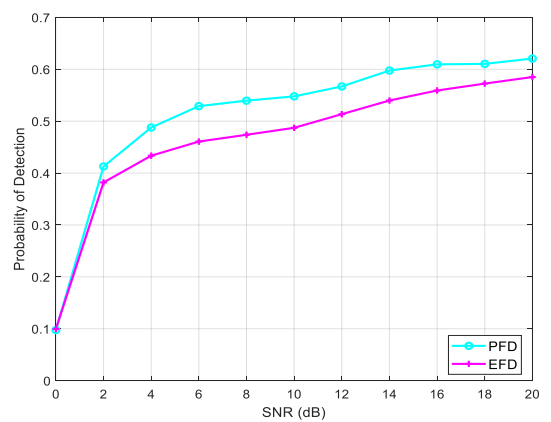

Figure 5. Probability of Detection (PD) versus SNR for PFD and EFD at L of 2 


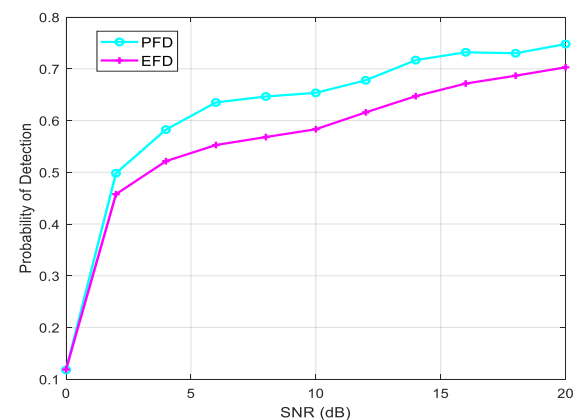

Figure 6. Probability of Detection (PD) versus SNR for PFD and EFD at L of 3

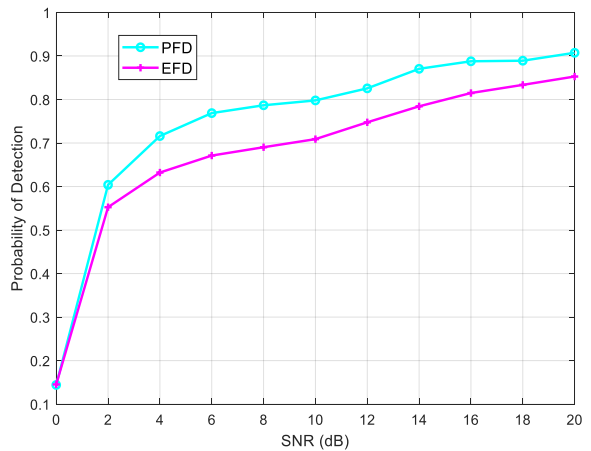

Figure 7. Probability of Detection (PD) versus SNR for PFD and EFD at L of 4

Figures 8 to 10 present SE versus SNR for PFD and EFD at different number of paths 'L' $(2,3,4)$. Fig. 8 depicts SE versus SNR for PFD and EFD at L of 2. SE values of 7.5579 and $6.7515 \mathrm{bps} / \mathrm{Hz}$ are obtained at SNR of $4 \mathrm{~dB}$ for PFD and EFD, respectively, while at SNR of $16 \mathrm{~dB}, 10.4056$ and $9.5991 \mathrm{bps} / \mathrm{Hz}$ are the corresponding SE values obtained for PFD and EFD. Fig. 9 depicts SE versus SNR at L of 3 for PFD and EFD. At SNR of $8 \mathrm{~dB}$, SE values of 11.2712 and $10.3034 \mathrm{bps} / \mathrm{Hz}$ are obtained for PFD and EFD, respectively, while the corresponding SE values obtained at SNR of $16 \mathrm{~dB}$ are 12.4867 and $11.5190 \mathrm{bps} / \mathrm{Hz}$ for PFD and EFD, respectively. SE versus SNR for PFD and EFD at L of 4 is presented in Fig. 10. At SNR of $8 \mathrm{~dB}, \mathrm{SE}$ values of 13.6786 and $12.5042 \mathrm{bps} / \mathrm{Hz}$ are obtained for PFD and EFD, respectively, while 15.1538 and $13.9793 \mathrm{bps} / \mathrm{Hz}$ are the corresponding SE values obtained for PFD and EFD, respectively, at SNR of $16 \mathrm{~dB}$. Figs. 8 to 10 revealed that, for all the number of paths, the proposed technique shows better bandwidth efficiency with higher SE when compared with EFD due to synchronization between licensed and unlicensed user signals that is not required for the PFD. Also, for both technique, SE increases as SNR and number of paths increase and this is as a result of higher signal strength at both SNR and number of paths increase. Therefore, the proposed technique shows greater bandwidth efficiency at higher SNR and number of paths.

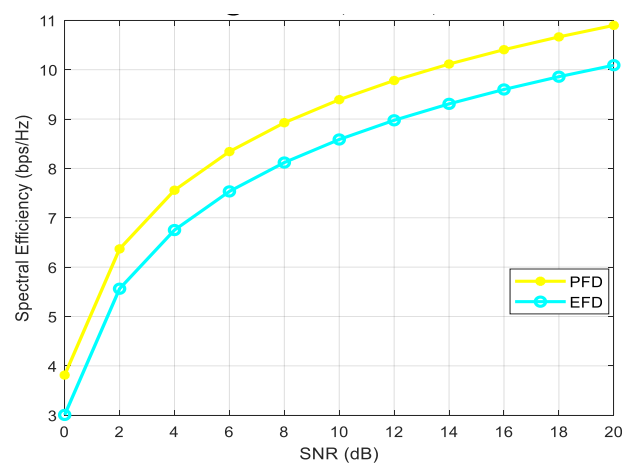

Figure 8. Spectral Efficiency (SE) versus SNR for PFD and EFD at L of 2 


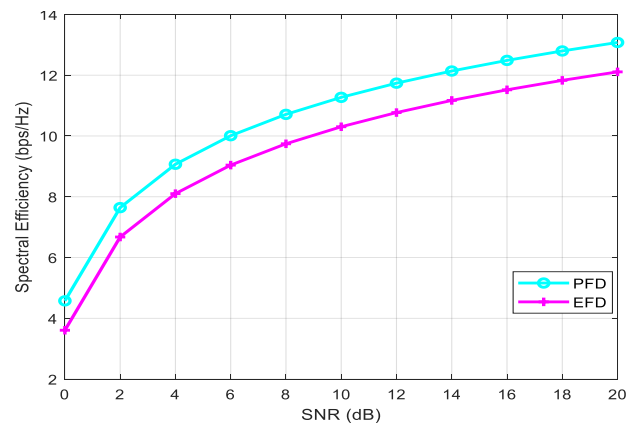

Figure 9. Spectral Efficiency (SE) versus SNR for PFD and EFD at L of 3

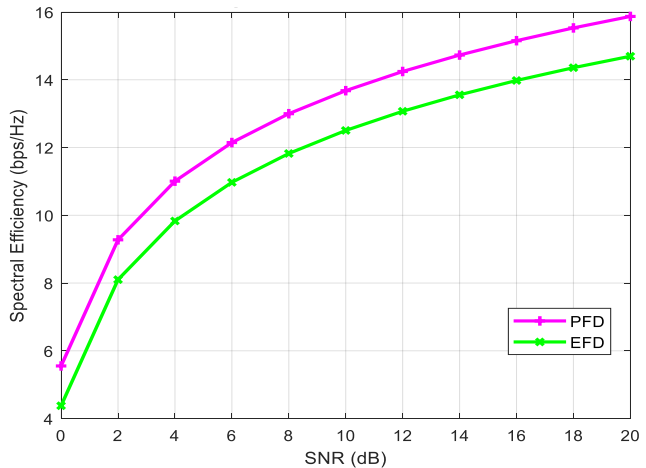

Figure 10. Spectral Efficiency (SE) versus SNR for PFD and EFD at L of 4

Figures 11 to 13 show ST versus SNR for PFD and EFD at different number of paths 'L' $(2,3,4)$. ST versus SNR is presented in Figure 11 for PFA and EFD at L of 2. At SNR of $4 \mathrm{~dB}$, ST values of 7.7638 and $14.2580 \mathrm{~s}$ are obtained for PFD and EFD, respectively, while 6.5620 and $11.9633 \mathrm{~s}$ are the corresponding ST values obtained at SNR of $16 \mathrm{~dB}$ for PFD and EFD, respectively. Fig. 12 depicts the ST values obtained against SNR for the two techniques at L of 3. The ST values obtained at SNR of $4 \mathrm{~dB}$ are 4.9512 and $9.1162 \mathrm{~s}$ for PFD and EFD, respectively, while the corresponding ST values at SNR of $16 \mathrm{~dB}$ are 4.1819 and $7.6444 \mathrm{~s}$ for PFD and EFD, respectively. The better sensing duration of PFD is due to autocorrelation used to obtain the feature of the received PU signal rather than windowing and Hilbert transform used in the EFD. Also, the proposed technique does not require synchronization, thereby reducing the hardware complexity leading to reduction of power consumption and sensing rate. Similarly, Figure 13 shows ST versus SNR for PFD and EFD at L of 4. ST values of 3.7163 and $6.8307 \mathrm{~s}$ are obtained at SNR of $4 \mathrm{~dB}$ for PFD and EFD, respectively, while at SNR of $16 \mathrm{~dB}, 3.1591$ and $5.7344 \mathrm{~s}$ are the corresponding ST values obtained for PFA and EFD. The results obtained reveal that ST decreases as number of paths and SNR increase due to increase in signal strength that enhances sensing rate.

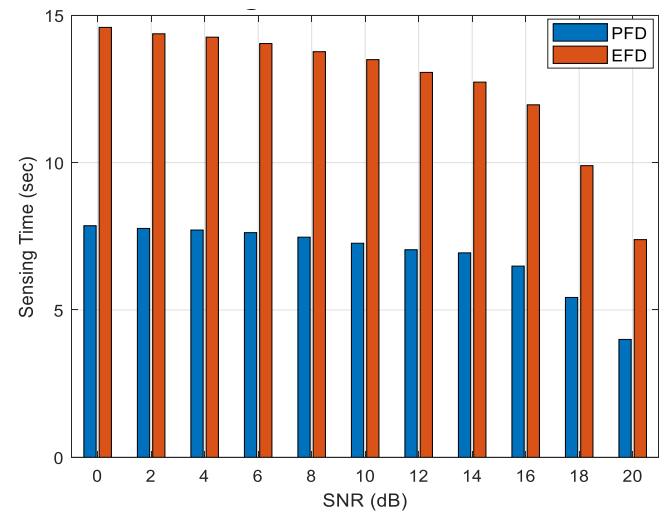

Figure 11. Sensing Time (ST) versus SNR for PFD and EFD at L of 2 


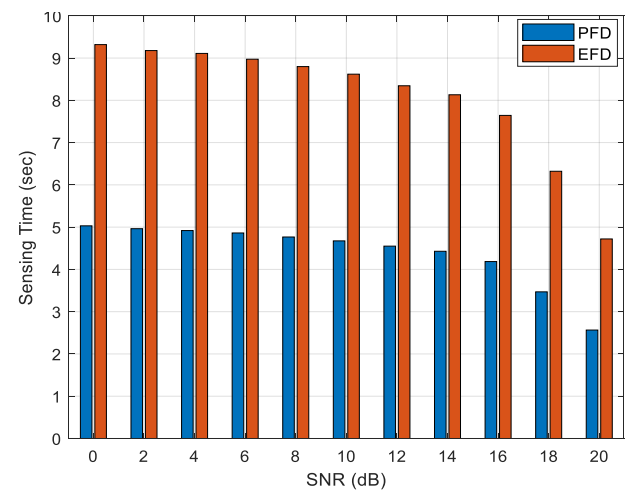

Figure 12. Sensing Time (ST) versus SNR for PFD and EFD at L of 3

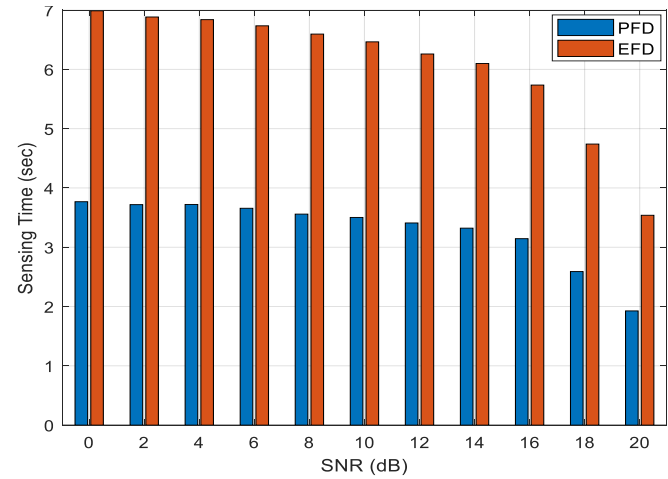

Figure 13. Sensing Time (ST) versus SNR for PFD and EFD at L of 4

\section{CONCLUSION}

This paper proposes an autocorrelation based white space detection in a multiple antenna energy harvesting enabled cognitive radio network. The multiple copies of PU signal are received using multiple antenna and combined using a modified Equal Gain Combiner (mEGC). The information and energy are obtained from the combined signal using antenna switching technique. The obtained energy is used to power a node, while the information obtained is used to determine the presence of white space. Autocorrelation is obtained and compared with the set threshold of zero to determine the idleness of the spectrum. The PDF of the combined Rayleigh and Log-Normal fading channel is obtained and used as fading distribution for the proposed technique. The technique is simulated and evaluated using PD, SE and ST by comparing with EFD technique. The results obtained show that the proposed technique performs better than the EFD due to higher PD and SE with lower PT values. The better performance of the PFD technique with higher PD is due to combining nature of the PU signals in the proposed technique before obtaining autocorrelation and the better sensing duration of proposed PFD technique is due to autocorrelation used to obtain the feature of the received PU signal rather than windowing and Hilbert transform used in the EFD. Also, the proposed technique does not require synchronization, thereby reducing the hardware complexity leading to reduction of power consumption and sensing rate. The higher SE obtained in PFD is due to synchronization between licensed and unlicensed user signals that is not required. Also, the results obtained depict the fact that PD increases as number of paths increases but ST decreases as the number of paths increases. Therefore, the PFD proposed has been shown to have a better performance by having a higher PD and SE with lower ST than the PFD technique. The study shows the reduction in hardware complexity with increase in signal strength and can be implemented in wireless communication for signal detection. In the proposed technique, SUs were assumed to be stationary. Future research can focus on how to facilitate mobility of SUs. Also, $\mathrm{CH}$ was fixed in the proposed technique, future research can focus on how to select $\mathrm{CH}$ from SUs based on distance between SU and PU at any time. 


\section{REFERENCES}

[1] Z.K. Adeyemo, S. I. Ojo, R.O. Abolade and O. B. Oladimeji. Modification of a Square-Law Combiner for Detection in a Cognitive Radio Network, International Journal of Wireless and Microwave Technologies, $\mathbf{4}(2)$ : 32-45, 2019.

[2] R.O. Abolade, S.I. Ojo, I.A. Ojerinde, J. S. Adetunji, and A. T. Lawal. Modification of Maximal Ratio Combining Technique for Detection of Spectrum Hole in a Cognitive Radio Network, International Journal of Wireless and Microwave Technologies, 7(2): 9-21, 2020.

[3] S.S. Elsayed, F. T. Ibrahim and A. A. Amman. Spectrum access queving based scheme for prioritized cognitive radio network, International Journal of Wireless and Mobile Computing, 15(4):351-358, 2018.

[4] S. Meenakshi, C. Prakash and S. Nityananda. A brief review of cooperative spectrum sensing: issues and challenges, IEEE Transaction on Wireless Communication, 16(3):1-4, 2016.

[5] M. Faten, A. Wissam and W. Mohieddin. A Classification of the Spectrum Sensing Techniques for Cognitive Radio, International Journal of Recent Technology and Engineering, 8(4):11586-11593, 2019.

[6] M. Yang, L. Yuan, L. Xiaofeng and T. Wenyan, T. Cyclostationary Feature Detection Based Spectrum Sensing Algorithm under Complicated Electromagnetic Environment in Cognitive Radio Networks, Journal of Synergetic radio cooperative and collaborative Radio, 4(1): 35-43, 2015.

[7] S. Noor. Combined soft hard cooperative spectrum sensing in Cognitive Radio networks, Ph. D dissertations submitted to University of Windsor, pp 1-79, 2017.

[8] A. Nikhil, M. Rita. Cooperative spectrum sensing using hard-soft decision fusion scheme International Journal of open Information Technologies, 5(5):36-39, 2017.

[9] A. Srivastava, M. S. Gupta and G. Kaur (2020) Energy efficient transmission trends towards future green cognitive radio networks (5G): Progress, taxonomy and open challenges, Journal of Network and Computer Applications, vol. 168, https://doi.org/10.1016/j.jnca.2020.102760.

[10] A. Srivastava and G. Kaur (2021). Resource management for traffic imbalance problem in green cognitive radio networks, Physical Communication, vol. 48, https://doi.org/10.1016/j.phycom.2021.101437

[11] X. J. Dong, Y. B. Chen, G. Y. Yang, X. S. Pang and J. X. Yang. The optimization of improved energy detector in Cognitive Radio network, International Conference on Computer Information Systems and Industrial Applications, Yunnan Minzu University, pp 87-93, 2015.

[12] A. Rupali, S. Neelam and K. Himanshu. Performance improvement of energy detector in Cognitive Radio using SECP diversity Combining technique over fading channel, International Journal of Wireless and Mobile Computing, 18(2):167-174, 2019.

[13] K. P. Sushant. an anomaly detection technique-based intrusion detection system for wireless sensor network, International Journal of Wireless and Mobile Computing, 17(4):323-333, 2019.

[14] Z. K. Adeyemo and I. A. Ojedokun. EGC Receiver using Single RF Chain and Single MF over Composite Rayleigh and Rician Fading Channels, Journal of Engineering and Applied Sciences, 9(7): 992-994, 2014.

[15] P. Komal and D. Tanuja. Review on: spectrum sensing in Cognitive Radio using multiple antenna, International Journal of Innovative Science, Engineering and Technology 3(4):313-318, 2016.

[16] K.D. Sanjay, W. Isaac, G. Nitin and J. Rishabh. Optimal Secondary Users Selection for Cooperative Spectrum Sensing in Cognitive Radio Networks, IEEE Globecom Workshop, United Arab Emirates, PP 4-9, 2018.

[17] O. O. Gevira. Design of an optimal eigen value-based spectrum sensing algorithm for Cognitive Radio, Master thesis submitted to University of Nairobi, pp 12-47, 2016.

[18] M. Jayanta, K. B. Deepak and K. S. Manoj. Cyclostationary Based Spectrum Sensing in Cognitive Radio: Windowing Approach, International Journal of Recent Technology and Engineering, 3(1): 95-99, 2014.

[19] F.K. Ojo, D. O. Akande and M. F. M. Salleh. An overview of RF energy harvesting and information transmission in cooperative communication networks, International Journal of Telecommunication Systems, Springer 7(2):14, 2018.

[20] Y. Yuanyuan, H. Sai and Y. Changchuan. Cooperative transmission in energy harvesting-based cognitive D2D networks, Wireless Network Springer Science and Business 5(2): 1-10, 2017.

[21] E. J. Shraddha, E. P. Deepak and E. S. Pawan. Eigen value Detection for Spectrum Sensing in Cognitive Radio Network over Nakagami Fading Channel, International Journal of Advanced Research in Electronics and Communication Engineering, 7(6): 1-4, 2018.

[22] G. R. Georg and E. S. C. Prema. Cyclostationary Feature Detection Based Blind Approach for Spectrum Sensing and Classification, Journal of Radio Engineering, 28 (1): PP 298-303, 2018.

[23] Z. Jingbo, R. Feng and L. Da. Maritime cognitive radio spectrum sensing based on multi-antenna cyclostationary feature detection, International Journal of Electronics, Tailor and Francis group, doi:10.1080/00207217.2019.1692373, pp 1-17, 2019.

[24] P. S. Santar and S. C. Sharma. an improve cluster-based routing algorithm for energy optimisation in wireless sensor networks, International Journal of Wireless and Mobile Computing, 14(1):82-89, 2018.

[25] P. Elechi and P. O. Otasowie. Determination of Path Loss Exponent for GSM Wireless Access in Rivers State Using Building Penetration Loss, The Mediterranean Journal of Electronics and Communications, 11(1): 2-8, 2015. 


\section{BIOGRAPHY OF AUTHORS}
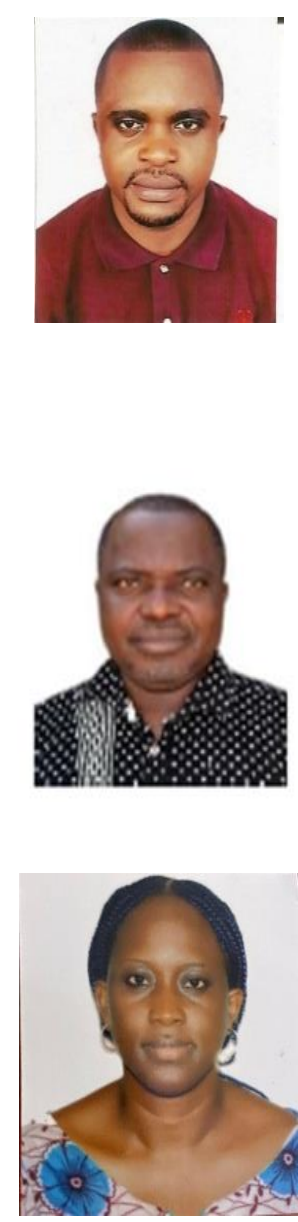

Rebeccah O. Omowaiye received her B. Tech and M. Tech degrees in Electronic and Electrical Engineering in 2005 and 2020, respectively, from Ladoke Akintola University of Technology (LAUTECH), Ogbomoso, Nigeria. He is a registered member of Council for the Regulation of Engineering in Nigeria (COREN). He is currently pursuing his Ph. $\mathrm{D}$ in communication Engineering. Her research interest is on signal processing and cooperative communications

Zachaeus K. Adeyemo obtained the B.Eng. and M. Eng. degrees in Electrical Engineering from University of Ilorin, Ilorin, Nigeria and his Ph.D. degree in Electronic and Electrical Engineering in 2009 from Ladoke Akintola University of Technology (LAUTECH), Ogbomoso, Nigeria. Prof. Adeyemo is a professor of Communication Engineering at the Department of Electronic and Electrical Engineering, LAUTECH, Ogbomoso. He is a member of the IEEE and registered engineer, Council for the Regulation of Engineering in Nigeria (COREN). His research interest is on wireless communications.

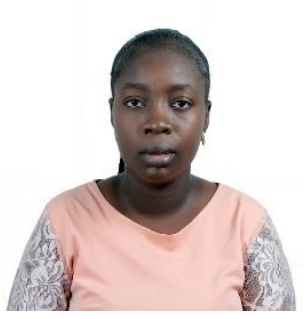

Oluwatobi O. Oyedokun received her B. Tech and M. Tech degrees in Electronic and Electrical Engineering in 2011 and 2020, respectively, from Ladoke Akintola University of Technology (LAUTECH), Ogbomoso, Nigeria. He is a member of Nigerian Society of Engineer (MNSE). He is currently pursuing his Ph.D in communication Engineering. Her research interest is on adaptive communication system and signal processing. 